\title{
Éditorial
}

\section{Après le sabre, le goupillon !}

Après une jeunesse insouciante, la revue Médecine Buccale Chirurgie Buccale (MBCB) se doit de devenir adulte et de trouver sa place (indexation) au sein de la littérature scientifique internationale.

Certes le chemin pour obtenir l'indexation est souvent tortueux et la décision de l'instance de référence échappe quelquefois à l'entendement. Néanmoins on doit reconnaitre que cette démarche a imposé très rapidement une nette évolution dans la présentation et la teneur des articles publiés dans les revues de notre spécialité. Toutefois, on a assisté à une dérive : l'impact factor occupant désormais une place prépondérante dans le CV, seules les revues référencées bénéficient de la sollicitude des auteurs. Ceci a également conduit à une augmentation exponentielle du nombre d'articles publiés sans obligatoirement observer une croissance proportionnelle des compétences et des connaissances cliniques de leurs auteurs. Toutefois, il faut bien reconnaitre que cette évolution a été bénéfique (rapport bénéfice/risque positif) pour les auteurs, les lecteurs et in fine pour les patients. Pour présenter un article, il faut donc s'astreindre à respecter les conseils aux auteurs (une nouvelle version sera prochainement disponible sur le site de la revue) ou tout simplement s'inspirer de la mise en page d'un article publié dans ce numéro. Enfin, dernièrement, certaines revues ont adopté deux nouvelles mesures : la déclaration obligatoire d'un conflit d'intérêt et le degré d'implication de chaque auteur dans l'étude et la rédaction de l'article. Après les objections suscitées récemment par l'attribution de certains Prix Nobel (conflits d'intérêt touchant quelques membres du jury, attribution du Prix Nobel à un responsable hiérarchique sans récompenser le principal acteur - cf. le livre. «Tout le monde doit connaitre cette histoire » de Jean-Claude Chermann et Olivier Galzi, Stock, Paris, 2009 -), il semble évident que ces deux mesures s'imposeront un jour à tous les auteurs. Il serait donc souhaitable que l'on s'imprègne d'ores et déjà de l'esprit de ces mesures qui appellent à un peu plus d'éthique.

Par ailleurs, vous n'êtes pas sans ignorer que l'Organisation internationale de Nominalisation vient de sacrifier sur l'autel de la mondialisation et de la modernité le terme art dentaire et de le remplacer par celui de médecine bucco-dentaire qui reflète effectivement l'évolution de notre activité. En effet, il y a bien longtemps que l'activité et les compétences du chirurgien dentiste ont dépassé le cadre de l'art dentaire. La médecine bucco-dentaire constitue un terme plus expressif et plus approprié que ceux de chirurgie dentaire et médecine dentaire qui donnent l'impression que le praticien tourne seulement autour de la dent. Toutefois, il faut reconnaitre que ce terme frise le pléonasme et, surtout, il sera difficile de trouver un nom au praticien qui se consacre à cette activité. Peut-être aurait-on pu explorer d'autres pistes? Par exemple, celle de la stomatologie. Dans son sens littéral actuel, c'est une « partie de la médecine qui traite des maladies de la bouche et des dents » (Petit Robert). Les médecins se sont appropriés ce terme à une époque où nous nous complaisions encore dans l'art dentaire. Pour décrire une activité quasi identique à celle du stomatologue, il fallait donc trouver un subterfuge. Mais pourquoi n'a-t-on pas choisi médecine buccale : le terme médecine pris dans son sens générique englobe la chirurgie, et le qualificatif buccal permet de définir notre champ d'activité ; était-il nécessaire d'abonder dans le détail superfétatoire en rajoutant dentaire comme si les dents n'étaient pas dans la cavité buccale? Le chirurgien (médecin) dentiste serait alors devenu un médecin buccal. De prime abord, ce terme heurte un peu l'oreille mais avec le temps, il se serait certainement imposé à l'instar d'autres termes comme médecin généraliste, médecin interniste... Vous allez me dire, et alors que serait devenue notre spécialité Médecine buccale Chirurgie buccale. Pas d'inquiétude, nous avons tout le temps d'y réfléchir, car le représentant de l'ADF, accessoirement de la France, responsable du Pôle de normalisation de l'ADF, sera président de l'ISO TC106/SC3 « Médecine bucco-dentaire Vocabulaire » la prochaine fois dans 25 ans environ! (cf. Info Dent 13 janvier 2010, 15-6). 